\title{
Analysis of the surface state of epi-ready Ge wafers
}

\author{
M. Gabás ，S. Palanco , S. Bijani , E. Barrigón ，C. Algora \\ I. Rey-Stolle , I. García , J.R. Ramos-Barrado
}

\begin{abstract}
A B S T R A C T
The surface state of Ge epi-ready wafers (such as those used on III-V multijunction solar cells) supplied by two different vendors has been studied using X-ray photoemission spectroscopy. Our experimental results show that the oxide layer on the wafer surface is formed by $\mathrm{GeO}$ and $\mathrm{GeO}_{2}$. This oxide layer thickness differs among wafers coming from different suppliers. Besides, several contaminants appear on the wafer surfaces, carbon and probably chlorine being common to every wafer, irrespective of its origin. Wafers from one of the vendors show the presence of carbonates at their surfaces. On such wafers, traces of potassium seem to be present too.
\end{abstract}

\section{Introduction}

Germanium is a substrate of rising importance in the field of III-V compound semiconductor epitaxy. Nowadays, Ge wafers are mostly used in the manufacture of triple-junction solar cells ( $\sim 95 \%$ of the market) though there are other emerging applications involving epitaxial growth such as thermophotovoltaic devices, photodetectors, light emitting diodes (LEDs), magnetoresistive sensors and even high electron mobility transistors (HEMTs) [1]. In the case of multijunction solar cells, and in most of the aforementioned applications, the final architecture of the device consists of a stack of epitaxially grown layers of different III-V materials, with varying thickness and doping levels. As in any layered electronic device, the interface between two films is a critical part since existing defects and impurities can profoundly affect the final device performance. In this respect, a key interface for any epitaxially grown electronic device is precisely the first one, i.e. that between the substrate and the epilayers grown onto it, since problems at that surface typically propagate and impact the performance of the whole device. Accordingly, a good epitaxy starts with a thorough understanding of the substrate surface. The presence of oxides, morphological imperfections, contaminants or adsorbates can have a key role in the formation of defects during growth of any layer onto these substrates [2]. Thereby, any characterization technique able to provide insight about the state of the surface prior to epitaxial growth is of utmost interest for the grower, in order to understand and justify the achievement of good (or poor) semiconductor structures and devices.

Current optoelectronic devices based on Ge demand the socalled epi-ready wafers, i.e. substrates with surfaces prepared to be directly used in an epitaxial reactor without any further cleaning [3]. The exact treatment to transform a Ge surface into an epi-ready surface is not disclosed but, in essence, consists of a set of cleaning steps aimed to remove metal contamination and to create carbon and oxide layers which are readily removable by in situ treatment in the growth reactor.

X-ray photoemission spectroscopy (XPS) is a widely extended surface analysis technique that yields information on the first few nanometers of a film. Its abilities range from the identification of any contaminant or residues at the sample surface, to the analysis of the orientation of adsorbed molecules or clusters into a particular surface. Photoelectron Spectroscopy (PES) of core levels is a sensitive probe of the local chemical environment around the emitting atom and its abilities have been proven for example in the photovoltaic field [4-6].

Accordingly, we present the study that we have carried out on the surface state of commercial Ge epi-ready wafers coming from two different vendors, namely, A and B, by using the XPS technique. The goal of this work is to gain a better understanding of epi-ready surfaces of commercial germanium wafers, in order to set the basis to explain differences in growth results often encountered in III-V on Ge epitaxy [ 2 and references therein]. This way, more efficient and reproducible $\mathrm{Ge}$-based optoelectronic devices can be achieved. To this end, we have identified the mean chemical state of Ge and searched for the presence of contaminants and residues on the asreceived wafer surface by using XPS technique. 


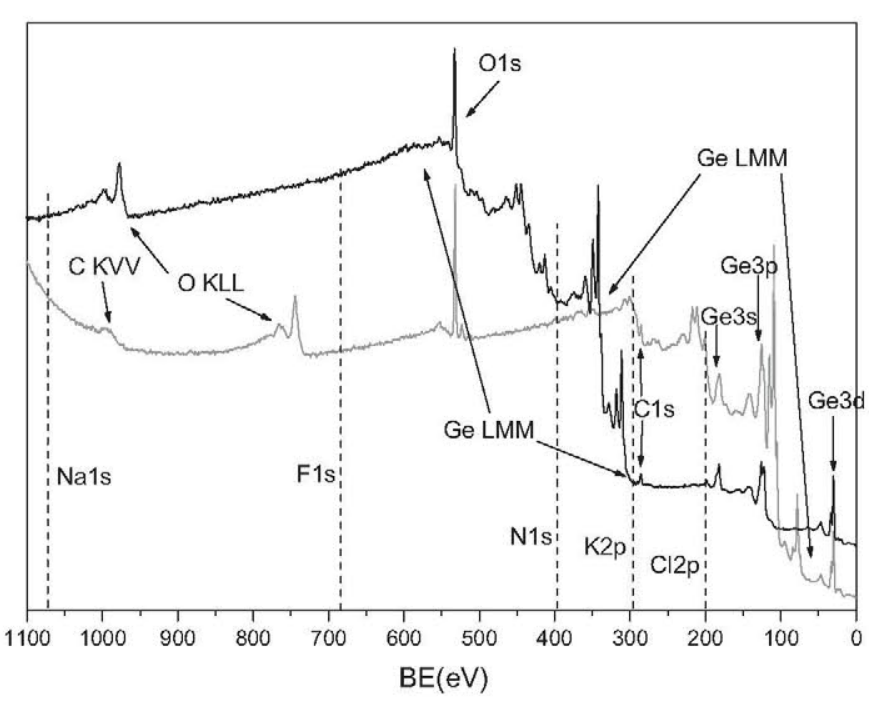

Fig. 1. Survey spectra of B wafers measured with both excitation sources: Mg Ko (grey line) and Al K $\alpha$ (black line). Vertical dashed lines mark the positions of the most intense core levels corresponding to several possible contaminants.

\section{Experimental}

Ge wafers used in this study were supplied by two different vendors, namely A (four wafers) and B (three wafers). The orientation of the substrates was $(100)$ with a miscut of $6^{\circ}$ towards the nearest (111) plane and they were doped with gallium (p-type) in the range $1 \times 10^{18}$ to $6 \times 10^{18} \mathrm{~cm}^{-3}$. The wafer from vendor A, i.e. Awafers (and correspondingly, for vendor B) show an average etch pit density lower than $500 \mathrm{~cm}^{-2}$, while B supplier guarantees no etch pits on its B-wafer surface.

The XPS system at the University of Málaga (Spain) was a Thermo Scientific Multilab 2000 spectrometer fitted with a dualanode X-ray source (Mg K $\alpha$ and $\mathrm{Al} \mathrm{K \alpha}$ with photon energies 1253.6 and $1486.7 \mathrm{eV}$, respectively), and a $110 \mathrm{~mm}$ hemispherical sector analyzer. Survey spectra and detailed core level spectra were measured using both X-ray sources. All the measurements were made on as-received wafers i.e. no $\mathrm{Ar}^{+}$sputtering cleaning was done. The core level spectra were fitted using the XPSPeak software package [7].

\section{Results}

\subsection{Survey spectra}

For a primary characterization, the wafer survey spectra were measured using, both $\mathrm{Mg} \mathrm{K} \alpha$ and $\mathrm{Al} \mathrm{K} \alpha$ sources. In that way, the presence of contaminants in small quantities at the surface of the wafers would not be masked by Auger peaks, since they would appear at different binding energies when the incident photon energy changes. In Fig. 1, the survey spectra of one of the wafers from B supplier are shown as measured with both excitation sources. Similar survey spectra were recorded for all the analyzed wafers. For the sake of clarity, the binding energy (BE) range has been limited to $1100 \mathrm{eV}$, thus, the Ge 2p core level (BE $\left.\left(\mathrm{Ge} 2 \mathrm{p}_{3 / 2}\right) \approx 1218 \mathrm{eV}\right)$ is absent in this figure. Besides the different inelastic background, the main differences between both spectra correspond to the positions of the Auger lines Ge LMM, O KLL and C KVV, the latter not appearing in the spectrum measured with the $\mathrm{Al} \mathrm{K} \alpha$ excitation source since it would be placed at a binding energy of $1223 \mathrm{eV}$, out of the range covered by Fig. 1. The peaks corresponding to $\mathrm{Ge} 3 \mathrm{p}, \mathrm{Ge} 3 \mathrm{~s}$ and $\mathrm{C} 1 \mathrm{~s}$ core levels are overlaid by the Auger Ge LMM spectrum when the Mg K $\alpha$ excitation source is used.

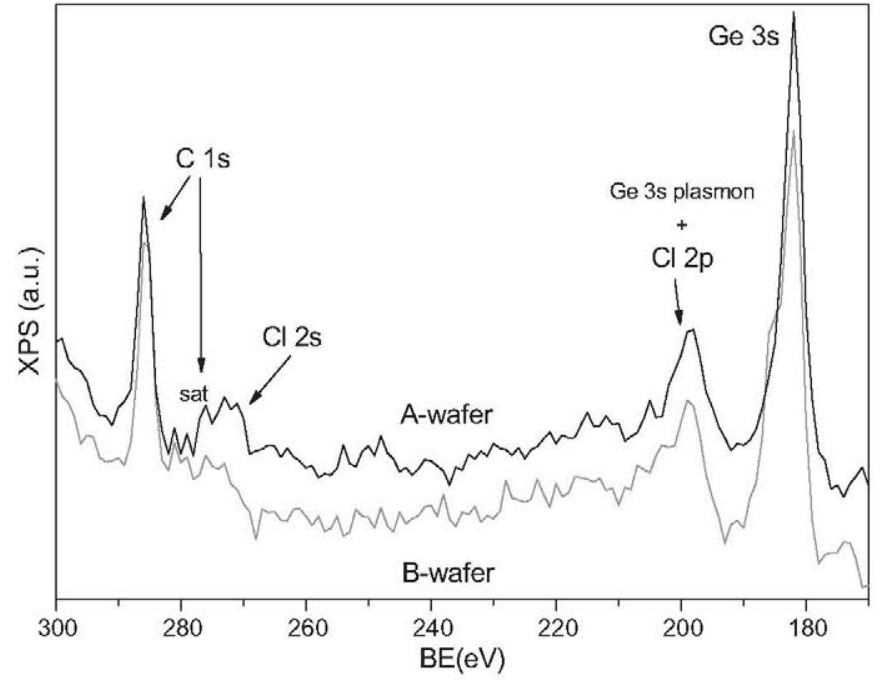

Fig. 2. Restricted binding energy area of the survey spectra of A and B-wafers mea-

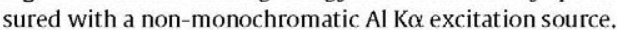

However, there is no such overlapping in the spectrum measured with the $\mathrm{Al} \mathrm{K} \alpha$ excitation source.

The asymmetric shape of Ge core levels and the presence of rather intense $O 1$ s and $O$ KLL signals, are a proof of the wafer surface oxidation. Irrespective of their origin, all the wafers show an oxidation layer at the topmost surface. Nevertheless, there are differences between wafers from different vendors. A-wafers seem to be less oxidized than wafers coming from B supplier, as will be shown when discussing Fig. 2.

Fingerprints of several contaminants ( $\mathrm{C}, \mathrm{N}, \mathrm{F}, \mathrm{Cl}, \mathrm{K}, \mathrm{Na}$ ) have been searched for on the wafer surfaces. Carbon presence is revealed through the $C 1$ s core level and the $C$ KVV Auger line, as it is expected in every sample surface exposed to air. Traces of fluorine, sodium or nitrogen were not found in any of the wafers since there are no signals of their most intense core levels, F $1 \mathrm{~s}(685 \mathrm{eV}), \mathrm{Na} 1 \mathrm{~s}$ $(1072 \mathrm{eV})$ and $\mathrm{N} \mathrm{1s}(398 \mathrm{eV})$, as it is shown in Fig. 1. The signal corresponding to $\mathrm{K} 2 \mathrm{p}_{3 / 2}$ core level has a binding energy of $292.9 \mathrm{eV}$, which lies very close to the $C 1$ s core level and to the Ge LMM Auger signal. It is thus not possible to elucidate from Fig. 1 the existence of potassium contamination on the surface of the wafers and this point will be revisited at the subsection discussing $C 1 \mathrm{~s}$ core levels. The presence of chlorine cannot be discarded after a first evaluation of survey spectra. There is a little and broad peak at $\sim 200 \mathrm{eV}$ which could be a signal of the $\mathrm{Cl} 2 \mathrm{p}$ core level (BE Cl $\left.2 p_{3 / 2}=199 \mathrm{eV}\right)$. However, in this binding energy area, it coincides with the first plasmon associated to the $\mathrm{Ge} 3$ s core level. The origin of that peak is thus uncertain.

In order to elucidate the presence of chlorine, a more detailed view of a reduced binding energy area of the survey spectra mea-

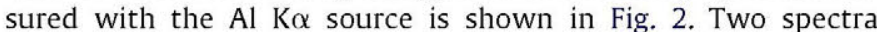
corresponding to A and B-wafers are presented. Ge 3 s and C 1s core levels appear in both picture extremes. The first one evidences the already mentioned differences in the mean Ge oxidation state, the peak associated to $\mathrm{B}$ wafer being more asymmetric. The feature on the high binding energy side of $\mathrm{Ge} 3$ s core level is asymmetric in both types of wafers. That points to the fact that it cannot be only a loss peak but, very probably, it is a convolution of the Ge 3 s first plasmon and the $\mathrm{Cl} 2 \mathrm{p}$ core level fingerprint. On the low binding energy side of the $\mathrm{C} 1 \mathrm{~s}$ core level peak, there is a broad feature with a complex structure. First of all, the presence of satellite lines should be taken into account since our X-ray source is nonmonochromatic. The binding energy distance of the first satellite line for an $\mathrm{Al} \mathrm{K \alpha}$ X-ray source is $9.8 \mathrm{eV}$. As shown in Fig. 2, this 


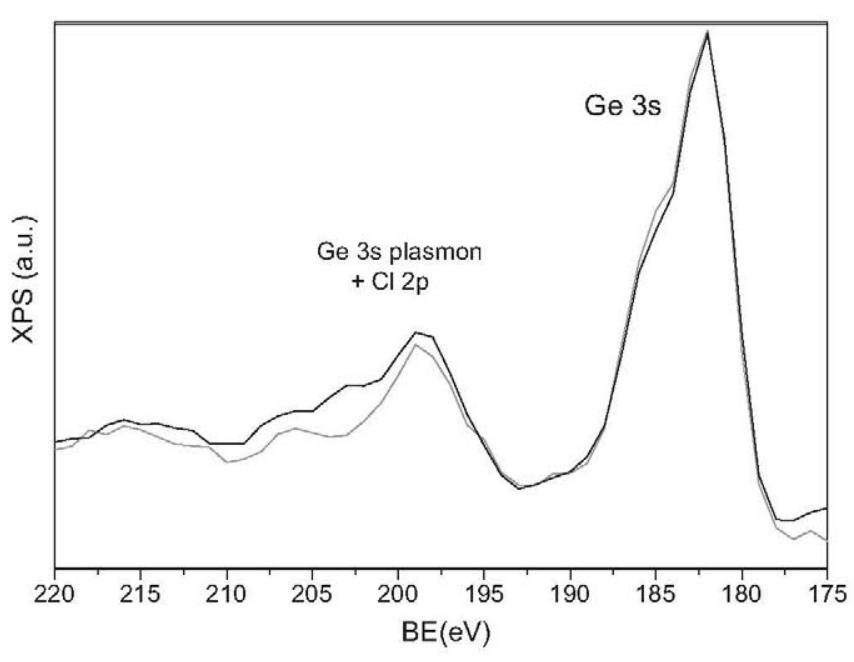

Fig. 3. Restricted binding energy area of two B-wafers (gray and black lines) survey spectra measured with an $\mathrm{Al}$ K $\alpha$ excitation source.

distance corresponds to the binding energy position, relative to $C$ $1 \mathrm{~s}$ core level peak, on the left part of that feature. However, it is too broad to be an only contribution. The next satellite line is placed $11.8 \mathrm{eV}$ away, on the right side of the main core level peak, but its intensity is half that of the first one. The $\mathrm{Cl} 2$ s core level has a binding energy of $271 \mathrm{eV}$, thus, its presence could be considered to explain the broad feature on the right side of the $\mathrm{C} 1 \mathrm{~s}$ core level peak, as it is indicated in Fig. 2.

Fig. 3, shows the partial survey spectra of two B-wafers with the same oxidation state, as demonstrated by Ge core levels shape. The features on the left side of the Ge 3 s core level are clearly asymmetric, supporting thus the conclusion that they are the convolution of the 1 st $\mathrm{Ge} 3$ s plasmon and the $\mathrm{Cl} 2 \mathrm{p}$ core level signal. Moreover, they show little differences on the shape and intensity. Since the plasmon structure should be identical for these wafers, the differences could account for variations in the chlorine quantity at the wafer surface. Therefore, the probable presence of chlorine as a contaminant on the studied wafer surfaces would be thus supported by several experimental findings. Its origin could be related to the wafer surface preparation, since several procedures would include the use of $\mathrm{HCl}$ during wafer cleaning [8-10].

\subsection{Ge core levels}

The Ge core levels have been studied in order to estimate the oxidation degree in the epi-ready wafers. As it has been already pointed out, B-wafers look more oxidized than A-wafers. The differences in the Ge mean oxidation state are presented in Fig. 4, where the Ge $2 \mathrm{p}_{3 / 2}$ spectra from A and B-wafers are depicted. The global signal has been deconvoluted into their components: metallic $\mathrm{Ge}$, $\mathrm{GeO}$ and $\mathrm{GeO}_{2}$. No other sub-oxides have been found in the wafers, according to what has already been described for wafers with a natural oxide layer [11]. No restrictions on the position or full width at half maximum (FWHM) of the different contributions were applied, except that the oxide peak FWHMs should be identical. After fitting, the values obtained for these parameters, summarized in Table 1, are quite similar for all the wafers studied. The mean peak energies of $\mathrm{GeO}$ and $\mathrm{GeO}_{2}$ are 1219.2 and $1220.9 \mathrm{eV}$, respectively, which are very similar to the values found in [12] for wafers exposed to cleanroom air. As it is evident in Fig. 4, the amount of oxidized $\mathrm{Ge}$ is larger in wafers B, which implies a thicker oxide layer. In Table 2, the relative quantities of the different components of the Ge $2 \mathrm{p}_{3 / 2}$ signal, as calculated from the area of the corresponding fit component, are reflected for every wafer. For B-wafers, more

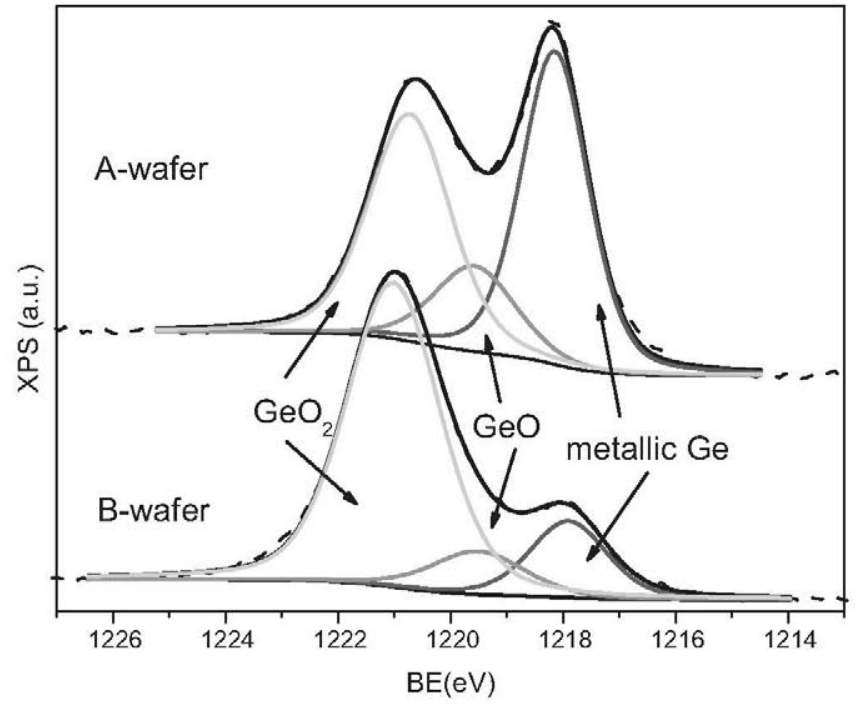

Fig. 4. Ge $2 p_{3 / 2}$ spectra of $A$ and $B$-wafers. Experimental data are dashed lines while fit result (black) and fit components (gray) are solid lines.

Table 1

Fit components of the $\mathrm{Ge} 2 \mathrm{p}_{3 / 2}$ and $\mathrm{Ge}$ 3d core level signals, with their corresponding mean binding energies and FWHM.

\begin{tabular}{|c|c|c|c|c|c|c|}
\hline & \multicolumn{2}{|l|}{$\mathrm{Ge}$} & \multicolumn{2}{|l|}{$\mathrm{GeO}$} & \multicolumn{2}{|l|}{$\mathrm{GeO}_{2}$} \\
\hline & $\mathrm{BE}(\mathrm{eV})$ & FWHM $(\mathrm{eV})$ & $\mathrm{BE}(\mathrm{eV})$ & FWHM(eV) & $\mathrm{BE}(\mathrm{eV})$ & FWHM $(\mathrm{eV})$ \\
\hline $\mathrm{Ge} 2 \mathrm{p}_{3 / 2}$ & 1217.88 & 1.54 & 1219.21 & 2.03 & 1220.88 & 2.03 \\
\hline $\mathrm{Ge} 3 \mathrm{~d}$ & 29.76 & 1.02 & 31.16 & 1.62 & 32.96 & 1.62 \\
\hline
\end{tabular}

Table 2

Relative intensity of the three fit components of the $\mathrm{Ge} 2 \mathrm{p}_{3 / 2}$ and $\mathrm{Ge} 3 \mathrm{~d}$ core level signals for all the analyzed wafers.

\begin{tabular}{|c|c|c|c|c|c|c|}
\hline & \multicolumn{2}{|l|}{$\mathrm{Ge}$} & \multicolumn{2}{|l|}{$\mathrm{GeO}$} & \multicolumn{2}{|l|}{$\mathrm{GeO}_{2}$} \\
\hline & Ge $2 p_{3 / 2}$ & $\mathrm{Ge} 3 d$ & $\mathrm{Ge} 2 \mathrm{p}_{3 / 2}$ & $\mathrm{Ge} 3 \mathrm{~d}$ & Ge $2 p_{3 / 2}$ & $\mathrm{Ge} 3 \mathrm{~d}$ \\
\hline A1-wafer & $48.39 \%$ & $85.71 \%$ & $16.41 \%$ & $5.09 \%$ & $35.20 \%$ & $9.20 \%$ \\
\hline A2-wafer & $47.73 \%$ & $83.29 \%$ & $17.60 \%$ & $6.48 \%$ & $34.67 \%$ & $10.23 \%$ \\
\hline A3-wafer & $42.46 \%$ & $82.23 \%$ & $14.09 \%$ & $5.91 \%$ & $43.45 \%$ & $11.80 \%$ \\
\hline A4-wafer & $29.71 \%$ & $74.72 \%$ & $11.46 \%$ & $6.99 \%$ & $58.83 \%$ & $18.27 \%$ \\
\hline B1-wafer & $12.62 \%$ & $60.49 \%$ & $9.50 \%$ & $7.32 \%$ & $77.87 \%$ & $32.19 \%$ \\
\hline B2-wafer & $1.51 \%$ & $42.48 \%$ & $5.72 \%$ & $6.17 \%$ & $92.77 \%$ & $51.35 \%$ \\
\hline B3-wafer & $12.19 \%$ & $63.31 \%$ & $8.71 \%$ & $6.94 \%$ & $79.10 \%$ & $29.75 \%$ \\
\hline
\end{tabular}

than $87 \%$ of the signal intensity corresponds to the oxides $\mathrm{GeO}_{2}$ and $\mathrm{GeO}$, while these components are about $53 \%$ of the total signal for type A-wafers, excluding the last one (A4), which was more oxidized than the rest of A-wafers. It is worth mentioning here that the kinetic energy of the photoelectrons that contribute to the signal of the Ge 2 p core level peak is very low, in that way, they carry out information from the topmost of the wafer surface. The take-off angle of the photoelectrons relative to wafer surface was $90^{\circ}$, thus, in this case, the mean escape depth (MED) of the photoelectrons would be the inelastic mean free path (IMFP). An estimation of this distance for $\mathrm{Ge} 2 \mathrm{p}$ outcoming photoelectrons [13] gives a value of $1 \mathrm{~nm}$ for the depth from which most of the signal (65\%) is emitted. The maximum sampling depth below the sample surface would be three times larger, but of course with a much lower contribution to the overall peak intensity from the buried layers. Our experimental findings allow to deduce that the germanium oxide layer in wafers $B$ would have a thickness larger than $1 \mathrm{~nm}$, while the estimated germanium oxide layer thickness for wafers A would be smaller, some tenths of nanometer. 


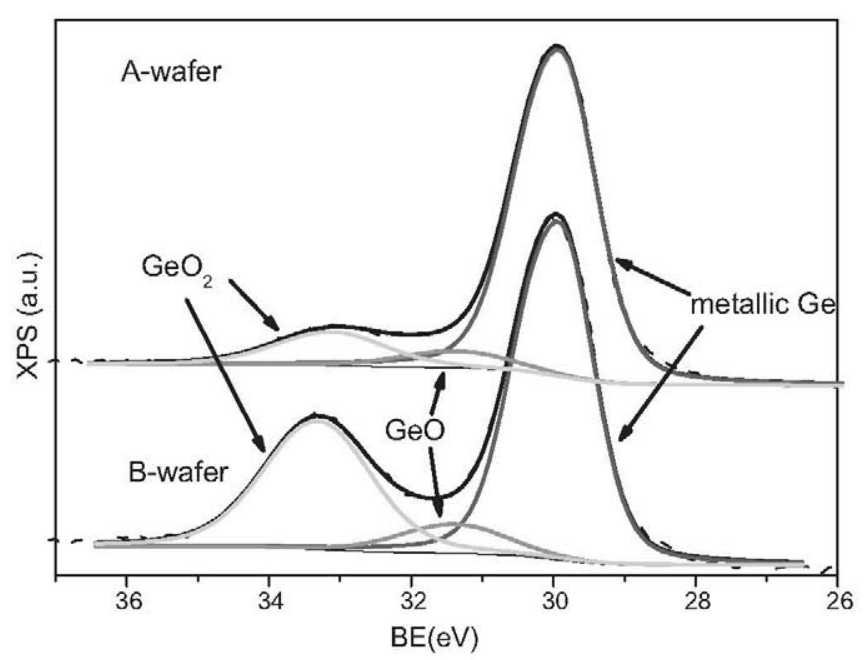

Fig. 5. Ge 3d spectra of A and B-wafers. Experimental data are dashed lines while fit result (black) and fit components (gray) are solid lines.

We have also studied the Ge 3d core level and the results corroborate the experimental findings described with the $\mathrm{Ge} 2 \mathrm{p}$ core level. There are three components: metallic $\mathrm{Ge}, \mathrm{GeO}$ and $\mathrm{GeO}_{2}$ for every $\mathrm{Ge} 3 \mathrm{~d}$ signal and the B-wafers are more oxidized than A-wafers. Fig. 5 shows the Ge 3d core level signals for the same wafers in Fig. 4. The kinetic energy of these photoelectrons is much larger than for the $\mathrm{Ge} 2 \mathrm{p}$ core level, thus, they mainly provide information from a more extended layer under the sample surface (IMFP $\approx 2.4 \mathrm{~nm}$ ). The enhanced intensity of the metallic Ge component when compared to what happens in Ge 2p core level, supports this fact. The fitting procedure was done imposing a spin-orbit splitting of $0.58 \mathrm{eV}$ and a branching ratio of $3: 2$ for the Ge $3 \mathrm{~d}_{5 / 2-3 / 2}$ doublet. A chemical shift of $1.4 \mathrm{eV}$ between the metallic and the $\mathrm{GeO}$ component was fixed to account the oxygen-induced core level shift per $\mathrm{Ge}-\mathrm{O}$ bond of $0.7 \mathrm{eV}$, as it is stated in [12] and [14]. As shown in Table 1, the chemical shift found for $\mathrm{GeO}_{2}$ oxide is $3.2 \mathrm{eV}$, which would imply an intermediate value for the oxygen-induced chemical shift per $\mathrm{Ge}-\mathrm{O}$ bond between $0.7 \mathrm{eV} \mathrm{[12]} \mathrm{and} 0.85 \mathrm{eV}$ [11]. The amount of metallic $\mathrm{Ge}$ is larger in the total intensity peak for this core level than for the Ge 2p core level. More than $80 \%$ for A wafers (except for the last one) and more than $60 \%$ for two B wafers and $42 \%$ for the B2 one. It has to be noted that, irrespective of the wafer origin, the quantity of $\mathrm{GeO}$ oxide found in the buried surface layer is almost equal for every wafer $(\sim 6 \%)$. This fact is probably related to the well-known stability of this oxide $[12,15]$.

\subsection{Oxygen core level}

O 1s core level spectra also evidence the different mean oxidation state of Ge wafers. The signal to background ratio grows for B-wafers, relative to what it is found on survey spectra of A-wafers (not shown). On these last wafers, the $\mathrm{O} 1 \mathrm{~s}$ signal is broader, which will lead us to the conclusion that, for A-wafers, the deconvolution of the signals needs an extra contribution besides to those of the two oxides seen in the Ge core level spectra (Fig. 6). All the contributions have the same FWHM, fixed at $1.45 \mathrm{eV}$. The two common contributions to the oxygen signal have mean binding energies of $531.2 \mathrm{eV}$ and $532.2 \mathrm{eV}$, which are very close to those reported for $\mathrm{GeO}$ and $\mathrm{GeO}_{2}, 531.3$ and $532.6 \mathrm{eV}$, respectively [12]. Their relative intensities follow the same tendency found in the Ge core level spectra for their counterparts. For all B-wafers, the ratio $\mathrm{GeO}_{2} / \mathrm{GeO}$ is larger than for A-wafers, as it is clearly seen in Fig. 6. This fact would be indicative of the more important oxidation in the first wafers. The third contribution that only appears in A-wafers has a

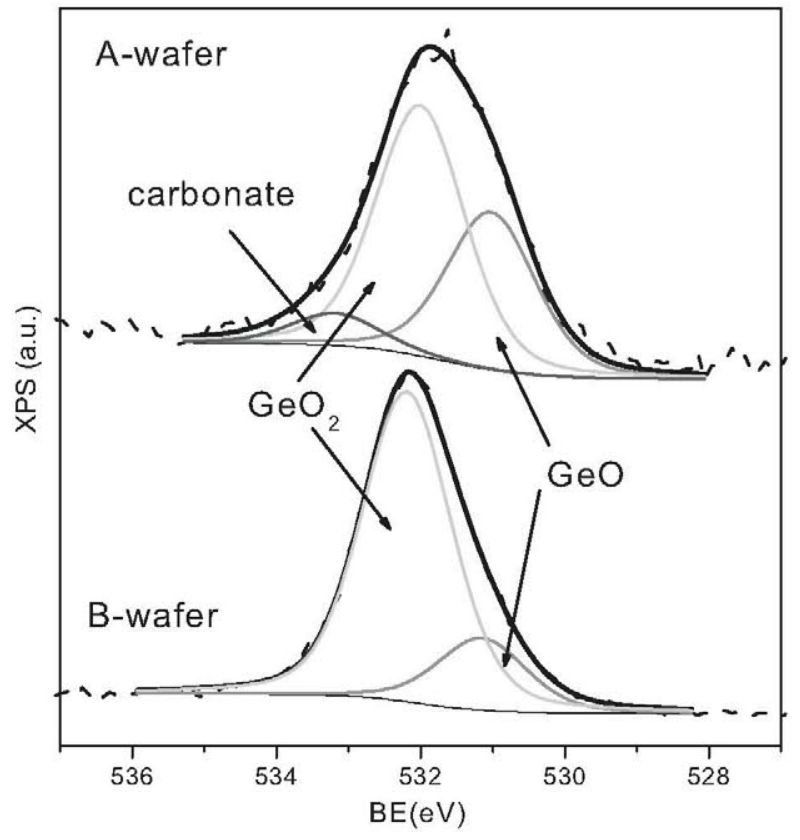

Fig. 6. $O$ 1s spectra of A and B-wafers. Experimental data are dashed lines while fit result (black) and fit components (gray) are solid lines.

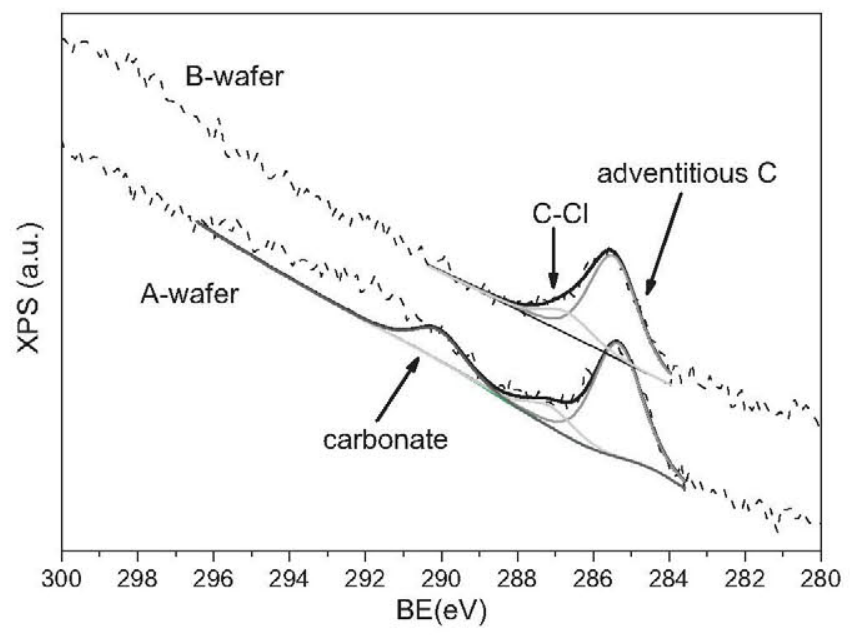

Fig. 7. $C$ 1s spectra of $A$ and $B$-wafers. Experimental data are dashed lines while fit result (black) and fit components (gray) are solid lines.

mean binding energy of $532.9 \mathrm{eV}$. The area of this contribution is between $7 \%$ and $20 \%$ of the total 01 s signal. These figures would be an estimation of the carbonates quantity on A-wafers surface. No other sub-oxides than $\mathrm{GeO}$ and $\mathrm{GeO}_{2}$ have been found in any $\mathrm{Ge}$ core level, thus the origin of this contribution must be related with the $C 1$ s core level signal appearing in all the survey spectra.

\subsection{1s core level}

In Fig. 7, the $\mathrm{C} 1 \mathrm{~s}$ core levels of both $\mathrm{A}$ and $\mathrm{B}$-wafers are depicted. The only restriction used during the fitting procedure concerned the peak FWHM, which was fixed to $1.5 \mathrm{eV}$. Low binding energy region is identical for all the wafers, with two contributions: one at $285.3 \mathrm{eV}$ and the smaller one at $286.7 \mathrm{eV}$. The first one is attributed to adventitious carbon, inherent to every sample exposed to air. To assign an origin to the second one, it must be taken into account that the total C 1s signal is very small and that the relative intensity of this contribution is less than $20 \%$ of the adventitious carbon 
contribution. Considering the probable presence of chlorine in these wafers, as it has been previously discussed when the survey spectra have been presented, we believe that a $\mathrm{C}-\mathrm{Cl}$ compound would be a possible origin of this contribution. The A-wafers show a broad feature in the high-energy spectra region, which is not present in the B-wafers spectra. It extends from 289 to $296 \mathrm{eV}$. A carbonate contribution, placed at $\sim 290 \mathrm{eV}$, would be consistent with the $\mathrm{O} 1 \mathrm{~s}$ spectra of these A-wafers, where a third contribution on the high binding region of $\mathrm{O} 1 \mathrm{~s}$ core level was found. Thus, the assignation of the third component origin in both, $\mathrm{O} 1 \mathrm{~s}$ and $\mathrm{C} 1 \mathrm{~s}$ core level spectra, for the A wafers has been made to a carbonate formed on the wafer surface. Finally, in these wafer spectra, there is still a small photoelectron intensity in the 292-296 eV energy range that remains unexplained. In this region, the doublet corresponding to the K 2 p core level has a very strong signal. This could be a possible source for that signal but further experiments are necessary to confirm the presence of potassium on these wafer surfaces.

\section{Conclusions}

XPS has demonstrated again its power as a tool for characterization and diagnosis at initial steps during the growth of III-V semiconductors on Ge substrates. The XPS analysis of germanium epi-ready wafers from two different vendors has demonstrated that, irrespective of the wafer origin, all the analyzed wafers have an oxide layer and show the presence of carbon and, probably, chlorine at their surfaces. No trace of fluorine, sodium nor nitrogen, which are typical remains after cleaning process of commercial wafers, was found. The variations detected between wafers from same vendor are negligible, but the comparison of the spectra of two wafers from different vendors shows evident differences. Wafers from vendor $B$ are more oxidized than those coming from vendor A, with an oxide layer that extends up to more than $1 \mathrm{~nm}$ in the first case whereas in second case it is some tenths of nanometer. The elimination of this oxide layer is not a difficult task by means of an in situ treatment within the growth reactor. However, A-wafers seem to be contaminated by some carbonate on their surfaces. The global analysis of the XPS data indicates that the presence of carbonates seems to inhibit the oxide layer growing. Therefore, the removal of these carbonates is an additional procedure to overcome prior to the epitaxial growth. The assertion of the presence of potassium on the type A wafers surface would need further experimental work. The identification of the contaminants at these epi-ready Ge-wafer surfaces constitutes the first step to asses their impact on Ge-based optoelectronic devices epitaxy.

\section{Acknowledgments}

This work has been supported by the Spanish Ministerio de Educación y Ciencia with the CONSOLIDER-INGENIO 2010 program by means of the GENESIS FV project (CSD2006-004). The Spanish Ministerio de Ciencia e Innovación has also contributed with the project TEC2010-16700, and the Spanish Ministerio de Economía y Competitividad with the projects TEC2011-28639-C0201 and -02, IPT-2011-1441-920000 and IPT-2011-1408-420000, as well as the Comunidad de Madrid under the NUMANCIA II programme (S2009/ENE1477). Fruitful discussions with Prof. E. Rodríguez-Castellón are gratefully acknowledged.

\section{References}

[1] B. Depuydt, A. Theuwis, I. Romandic, Germanium: from the first application of Czochralski crystal growth to large diameter dislocation-free wafers, Materials Science in Semiconductor Processing 9 (2006) 437-443.

[2] I. Rey-Stolle, E. Barrigón, B. Galiana, C. Algora, Analysis of germanium epiready wafers for III-V heteroepitaxy, Journal of Crystal Growth 310 (2008) $4803-4807$.

[3] K. Dessein, Role of the germanium substrate manufacturer in the CPV market, Presentation given at 2007 Marburg Workshop on Concentrating Photovoltaic Optics and Power, downloadable at http://www.concentrating pv.org/marburg2007/papers.html.

[4] P. Singh, S.M. Shivaprasad, M. Lal, M. Husain, Angle-dependent, XPS analysis of silicon nitride film deposited on screen-printed crystalline silicon solar cell, Solar Energy Materials \& Solar Cells 93 (2009) 19-24.

[5] M. Gabás, N.T. Barrett, J.R. Ramos-Barrado, S. Gota, T.C. Rojas, M.C. LópezEscalante, Chemical, Electronic interface structure of spray pyrolysis deposited undoped and $\mathrm{Al}$-doped $\mathrm{ZnO}$ thin films on a commercial $\mathrm{CZ}-\mathrm{Si}$ solar cell substrate, Solar Energy Materials \& Solar Cells 93 (2009) 1356-1365.

[6] K. Fostiropoulos, M. Rusu, Engineering of hybrid interfaces in organic photovoltaic devices, Solar Energy Materials \& Solar Cells 95 (2011) 1489-1494.

[7] http://public.wsu.edu/ scudiero/index.html.

[8] H. Okumura, T. Akane, S. Matsumoto, Carbon contamination free Ge(100) surface cleaning for MBE, Applied Surface Science 125 (1998) 125-128.

[9] J.S. Hovis, R.J. Hamers, C.M. Greenlief, Preparation of clean and atomically flat germanium (001) surfaces, Surface Science 440 (1999) L815-L819.

[10] S. Sun, Y. Sun, Z. Liu, D,--Ick Lee, S. Peterson, P. Pianetta, Surface termination and roughness of $\mathrm{Ge}(100)$ cleaned by $\mathrm{HF}$ and $\mathrm{HCl}$ solutions, Applied Physics Letters 88 (2006) 1-3, 21903.

[11] D. Schmeisser, R.D. Schnell, A. Bogen, F.J. Himpsel, D. Rieger, G. Landgren, J.F. Morar, Surface oxidation states of germanium, Surface Science 172 (1986) 455-465.

[12] K. Prabhakaran, T. Ogino, Oxidation of Ge(100) and Ge(1 11 ) surfaces: an UPS and XPS study, Surface Science 325 (1995) 263-271.

[13] http://www.lasurface.com/.

[14] O. Renault, L. Fourdrinier, E. Martínez, L. Clavelier, C. Leroyer, N. Barrett, C.Crotti, High-resolution photoelectron spectroscopy of $\mathrm{Ge}$-based $\mathrm{HfO}_{2}$ gate stacks, Applied Physics Letters 90 (2007) 1-3, 052112

[15] K. Prabhakaran, F. Maeda, Y. Watanabe, T. Ogino, Distinctly different thermal decomposition pathways of ultrathin oxide layer on Ge and Si surfaces, Applied Physics Letters 76 (2000) 2244-2246. 